\title{
Recent Transposition of an LTR-Retrotransposon in the Gene Coding for $S$ Receptor Kinase is Responsible for a Novel Self-Compatible Phenotype of Radish (Raphanus Sativus L.)
}

\section{So-Hyeon Bong}

Chonnam National University

Ganghee Cho

Chonnam National University

Dong-Seon Kim

FarmHannong Co Ltd

Sunggil Kim ( $\nabla$ dronion@jnu.ac.kr)

Chonnam National University https://orcid.org/0000-0001-8555-2995

\section{Research Article}

Keywords: Radish (Raphanus sativus L.), Self-incompatibility, S haplotype, self-compatible mutant, Duplicate SRK genes

Posted Date: September 23rd, 2021

DOI: https://doi.org/10.21203/rs.3.rs-884506/v1

License: (9) This work is licensed under a Creative Commons Attribution 4.0 International License. Read Full License 


\section{Abstract}

Self-incompatibility $(\mathrm{SI})$ responses of radish (Raphanus sativus L.) are determined by two tightly linked genes encoding an S receptor kinase (SRK) and an S-locus cysteine-rich protein/S locus protein 11 (SCR/SP11), respectively. A radish showing an almost self-compatible (SC) phenotype was identified in this study. Inheritance patterns showed that this SC phenotype was dominant over an SI phenotype. In addition, this SC phenotype co-segregated with an Shaplotype in an $\mathrm{F}_{2}$ population. This $\mathrm{SC}$ radish contained an $R S S-26$ haplotype in which duplicate $S R K$-like genes were previously identified. Full-length sequences of two $S R K$-like genes of 18,133-bp and 6,200-bp in length were obtained from radish with the $R s S-26$ haplotype (designated as RSSRK-26-1 and RsSRK-26-2, respectively). Duplicate SCR/SP11-like genes were also identified in the radish with the RsS-26 haplotype. Phylogenetic analyses indicated that both duplicate $S R K$-like and SCR/SP11-like genes were closely related to other known SRK and SCR/SP11 genes, respectively. No critical mutation was found in the coding region of SRK-like or SCR/SP11-like gene. However, a 4,146-bp intact LTR-retrotransposon was identified in the third intron of RsSRK-26-1 of the SC radish. Interestingly, this LTR-retrotransposon was not detected in three other breeding lines containing the same RsS-26 haplotype. Except for this LTR-retrotransposon, only two single nucleotide polymorphisms (SNPs) were identified in intronic regions between normal and mutant RsSRK-26-1 alleles. While normal transcription was observed for radish showing RsSRK-26-1 and SI phenotypes in these three breeding lines, no transcript of RsSRK-26-1 was detected in the SC radish, suggesting that recent transposition of an LTR-retrotransposon in the RsSRK-26-1 gene might be responsible for the SC phenotype of radish.

\section{Introduction}

Radish (Raphanus sativus L.) is one of the most important root vegetables in East Asian countries such as China, Japan, and Korea. As a cross-pollinated crop, radish shows hybrid vigor. Its $F_{1}$ hybrid varieties that harness such hybrid vigor have been developed (Curtis 2011). Self-incompatibility (SI) is defined as inhibition of pollen tube growth in pistils during self-pollination. It has been commercially used for the production of $F_{1}$ hybrid seeds in radish (Singh et al. 2001). SI systems are widespread in plants. They can promote outcrossing and increase genetic diversity. More than 100 families and almost $40 \%$ of angiosperms have adopted SI systems (Igic et al. 2008).

Generally, a single locus initially named as $S$ (Sterility) is involved in SI responses. Two tightly linked genes consisting of male and female determinants of SI are positioned in the Slocus. Nucleotide sequences of these two SI determinants are highly polymorphic and multi-allelic. Since at least two genes are present in the Slocus, combinations of these genes can lead to Shaplotypes (Stone and Goring 2001; Watanabe et al. 2012). Types of SI systems are largely categorized into heteromorphic and homomorphic $\mathrm{SI}$. In the case of heteromorphic SI, cross-pollination is possible between different floral morphs. Candidate genes responsible for distyly have been reported in Primula vulgaris (Li et al. 2016) and Fagopyrum esculentum (Yasui et al. 2012). 
Homomorphic SI systems are generally classified into gametophytic SI (GSI) and sporophytic SI (SSI) depending on inheritance patterns of SI phenotypes. Such inheritance patterns are determined by genotypes of haploid pollen in GSI and diploid pollen parents in SSI (Silva and Goring 2001; Muñoz-Sanz et al. 2020). GSI systems based on RNase and S-locus F-box proteins have been extensively studied in Solanaceae, Plantaginaceae, and Rosaceae families (Franceschi et al. 2012). The Papaver system based on programmed cell death has been mainly studied in poppy (Wheeler et al. 2009). Meanwhile, SSI has been extensively studied in Brassicaceae including radish (Sobotka et al. 2000; Takayama and Isogai 2003; Tantikanjana et al. 2010; Watanabe et al. 2012).

Two tightly linked genes encoding $S$ receptor kinase (SRK) and Slocus cysteine-rich protein (SCR)/S locus protein 11(SP11) or SP11/SCR have been revealed as female and male determinants of SSI, respectively (Schopfer et al. 1999; Takasaki et al. 2000; Takayama et al. 2000). Another gene encoding $S$ locus glycoprotein (SLG) has been identified in the Slocus, although its precise role remains uncertain (Nasrallah et al. 1985; Watanabe et al. 2012). Sequences of $S L G$ and $S$ domain of SRK genes are highly homologous within the same Shaplotypes (Sato et al. 2002; Lim et al. 2002; Okamoto et al. 2004). Based on sequence diversity of SLG and SRK genes, Shaplotypes are classified into class I and class II (Nasrallah et al. 1991; Sato et al. 2002). Generally, Shaplotypes of class I are dominant over those of class II and small noncoding RNAs and DNA methylation are involved in such dominance relationships (Tarutani et al. 2010; Yasuda et al. 2016).

SI was first used in production of $F_{1}$ hybrid seeds of cabbage in 1940s by Japanese seed companies. Since then, $\mathrm{SI}$ has been exploited for economical production of $\mathrm{F}_{1}$ hybrid seeds of radish and Brassica crops (Muñoz-Sanz et al. 2020). To implement SI systems in radish $\mathrm{F}_{1}$ hybrid breeding programs, specific $S$ haplotypes of parental lines should be identified to avoid cross-incompatibility between parental lines. At least 35 Shaplotypes have been identified in radish (Haseyama et al. 2018). More than 50 and $100 S$ haplotypes have been identified in Chinese cabbage (Nou et al. 1993) and cabbage (Ockendon 2000), respectively.

For efficient identification of $S$ haplotypes, polymerase chain reaction-restriction fragment length polymorphism (PCR-RFLP) methods have been developed for radish and Brassica crops (Brace et al. 1993, 1994; Nishio et al. 1994, 1996, 1997; Sakamoto et al. 2000; Lim et al. 2002). Although PCR-RFLP is more efficient than laborious pollination tests, it has pitfalls such as complicated band patterns of digested PCR products and difficulty in specific PCR amplification of SLG or SRK genes due to the presence of multiple homologous SRK-like genes (Boyes et al. 1991; Kim and Kim 2018). To overcome these problems, a new Shaplotyping system has been developed based on SLL2 and SP6 genes positioned at borders of $S$ core regions and $31 S$ haplotypes have been identified from diverse breeding lines in our previous studies (Kim et al. 2016; Kim and Kim 2019).

Self-compatible (SC) mutants have been found naturally or induced by irradiation in crops normally showing SI phenotypes (Muñoz-Sanz et al. 2020). In some fruit tree species, such SC phenotypes are sometimes advantageous for better fruit set and yield (Claessen et al. 2019). A radish breeding line 
showing a SC phenotype was identified and the critical mutation responsible for the SC phenotype was revealed in this study. In addition, efficient application of this SC phenotype in radish breeding programs is discussed.

\section{Materials And Methods Plant materials}

A radish breeding line (JNUR1537) showing an SC phenotype was introduced from a seed company (Farm Hannong Co., Ltd., Republic of Korea). Detail pedigree of this breeding line is unknown. JNUR1537 was crossed with JNUR1123 to produce $F_{1}$ hybrids by hand-pollination. An $F_{2}$ population was produced by self-pollination of $F_{1}$ hybrids. Three breeding lines (DBRL294-2, DBR2085 and DBR2086) containing the RsS3 haplotype were used to compare SI phenotypes with JNUR1537. Shaplotypes of these three breeding lines have been identified in a previous study (Kim and Kim 2019). Evaluation of SI phenotypes were performed in greenhouses. Single plants were covered with mesh cages to prevent cross-pollination. No insect pollinator was introduced to mesh cages.

\section{PCR amplification, genome walking, and sequencing of PCR products}

Total genomic DNAs were extracted from leaf tissues using a cetyl trimethylammonium bromide (CTAB) method (Doyle and Doyle 1987). PCR amplification and sequencing of PCR products of $S L L 2, S P 6$, and $S R K$ genes of parental lines of the $F_{2}$ population were carried out following methods described in a previous study (Kim and Kim 2019). The detail protocol for high-resolution melting (HRM) analysis has been described in a previous study (Seo et al. 2020). SYTO ${ }^{\circledR 9}$ green fluorescent nucleic acid stain (Thermo Fisher Scientific, Waltham, MA, USA) was used as a dye. HRM peaks were obtained using a LightCycler ${ }^{\circledR} 96$ system (Roche Molecular Systems, Pleasanton, CA, USA). Primer sequences of HRM markers are shown in Supplementary Table 1.

To obtain full-length genomic DNA sequences of duplicated SRK homologs, long PCR and genome walking were performed. Long PCR reactions were carried out in $50 \mu \mathrm{L}$ reaction mixtures containing 0.25 $\mu \mathrm{g}$ DNA template, $5 \mu \mathrm{L}$ 10x PCR buffer, $1.0 \mu \mathrm{L}$ forward primer $(10 \mu \mathrm{M}), 1.0 \mu \mathrm{L}$ reverse primer $(10 \mu \mathrm{M}), 8.0$ $\mu \mathrm{L}$ dNTPs (10 mM each), and $0.5 \mu \mathrm{L}$ Taq polymerase (TaKaRa LA PCR ${ }^{\mathrm{TM}}$ Kit Ver. 2.1; Takara Bio, Shiga, Japan). Long PCR amplification was performed with 40 cycles of $98^{\circ} \mathrm{C}$ for $10 \mathrm{~s}$ and $68^{\circ} \mathrm{C}$ for $15 \mathrm{~min}$. Genome walking was performed using a Universal GenomeWalker kit (Takara Bio) according to the manufacturer's instructions. Total genomic DNAs used to construct genome walking libraries were isolated from young seedlings using a DNeasy Plant Mini Kit (QIAGEN, Valencia, CA, USA). All PCR products were visualized on $1.5 \%$ agarose gels after ethidium bromide staining. Subsequently, PCR products were purified using a QIAquick PCR Purification Kit (QIAGEN). Sequencing was performed by a specialized company (Macrogen, Seoul, Republic of Korea). 


\section{RNA extraction and reverse transcription polymerase chain reaction (RT-PCR)}

Total RNAs were extracted from floral buds using a RNeasy Plant Mini Kit (QIAGEN). cDNAs were synthesized using a cDNA synthesis kit (SuperScript ${ }^{\text {TM }}$ III first-strand synthesis system for RT-PCR, Invitrogen, Carlsbad, CA, USA). RT-PCR amplification was performed with an initial denaturation step at $94^{\circ} \mathrm{C}$ for $3 \mathrm{~min}$, followed by 35 cycles of $94^{\circ} \mathrm{C}$ for $30 \mathrm{~s}, 68^{\circ} \mathrm{C}$ for $30 \mathrm{~s}$, and $72^{\circ} \mathrm{C}$ for $2 \mathrm{~min}$. It was finished with a final 10 -min extension step at $72^{\circ} \mathrm{C}$. Primer sequences used in RT-PCR are listed in Supplementary Table 1. Radish tubulin sequence (Rs395780) identified from a draft genome sequence (Jeong et al. 2016) was used as a control.

\section{Construction of phylogenetic trees}

Genomic DNA, cDNA, or deduced amino acid sequences of SLL2, SP6, SRK, and SCR/SP11 genes were aligned using a BioEdit software (Hall 1999). Large gaps in alignments were removed using Gblocks program (Castresana 2000). Phylogenetic trees were produced using MEGA version X (Kumar et al. 2018) with a neighbor-joining method. Node support of the phylogenetic tree was assessed using 1,000 bootstrap replicates.

\section{Results}

\section{Discovery of an SC radish breeding line and inheritance patterns of the SC phenotype}

Radishes derived from JNUR1537 showed an almost SC phenotype. Compared with an SI radish accession (Fig. 1A), a large number of pods were formed in the SC radish without any insect pollinators (Fig. 1B). This SC plant was cross-pollinated with a SI breeding line (JNUR1123). $F_{1}$ hybrids showed the SC phenotype, indicating that the SC phenotype was dominant over the SI phenotype. Subsequently, an $F_{2}$ population was produced from self-pollination of $F_{1}$ hybrids. Ten individuals of the $F_{2}$ population were grown in a greenhouse. Each individual plant was separately covered with mech cages. SI phenotypes clearly segregated among $F_{2}$ plants (Table 1 ). 
Table 1

Number of seeds produced from 10 randomly selected $\mathrm{F}_{2}$ individuals grown in isolated greenhouses

\begin{tabular}{|lll|}
\hline Plant code & Number of seeds & Genotype of Shaplotypes \\
\hline F2-1 & 540 & RsS3 / RsS3 \\
\hline F2-2 & 693 & RsS3 / RsS32 \\
\hline F2-3 & 74 & RsS32 / RsS32 \\
\hline F2-4 & 2,330 & RsS3 / RsS32 \\
\hline F2-5 & 1,483 & RsS3 / RsS32 \\
\hline F2-6 & 174 & RsS3 / RsS3 \\
\hline F2-7 & 1,012 & RsS3 / RsS32 \\
\hline F2-8 & 576 & RsS3 / RsS3 \\
\hline F2-9 & 86 & RsS32 / RsS32 \\
\hline F2-10 & 1,086 & RsS3 / RsS32 \\
\hline
\end{tabular}

To test whether the SC phenotype was related to $S$ haplotypes, specific Shaplotypes of both SC and SI parental lines were identified using an $S$ haplotyping system developed in a previous study (Kim and Kim 2019). From the SI parent, novel sequences of SLL2, SP6, and SRK genes closely related to RsSLL2-25, $R S S P 6-8$, and RSSRK9, respectively (Supplementary Figs. 1, 2, 3), were isolated. These novel allele sequences were designated as RSSLL2-31, RSSP6-26, and RSSRK21, respectively. Their nucleotide sequences were deposited into GenBank under accession numbers of MZ383797, MZ383798, and MZ383799, respectively. Since RSSRK21 was clustered with class II SRK genes (Supplementary Fig. 3), RsSK21 was considered as a member of class II SRK genes. The new class II Shaplotype consisting of three novel sequences was designated as RsS32. Nucleotide sequences of both SLL2 and SP6 genes in the SC parent were identical to those of the class II RsS3 haplotype. As expected, a mixture of duplicate SRK-like genes was amplified. In a previous study (Kim and Kim 2019), closely related duplicate SRK-like genes have also been detected in the RsS3 haplotype.

For efficient genotyping of $S$ haplotypes of $\mathrm{F}_{2}$ individuals, two HRM markers were developed based on polymorphic sequences of SLL2 and SP6 alleles of parental lines, respectively (Supplementary Fig. 4). After genotyping $F_{2}$ seedlings, five individuals of each genotype were separately grown in cages. While SC phenotypes were observed in both homozygous RsS3 and heterozygous individuals, few pods and seeds were formed in homozygous RsS32 individuals (Table 2). In addition, SI phenotypes of $10 \mathrm{~F}_{2}$ individuals previously grown in isolated greenhouse were matched with genotypes of $S$ haplotypes (Table 
1). These results implied that the SC phenotype was probably related to $S$ core genes such as $S R K$ and SCR/SP11.

Table 2

Numbers of pods and seeds produced from $\mathrm{F}_{2}$ individuals representing three genotypes of $S$ haplotypes

\begin{tabular}{|c|c|c|c|}
\hline Genotype & Plant code & Number of pods & Number of seeds \\
\hline \multirow[t]{6}{*}{ RsS3 / RsS3 } & $\mathrm{A} 1$ & 328 & 844 \\
\hline & $\mathrm{A} 2$ & 115 & 136 \\
\hline & A3 & 232 & 257 \\
\hline & A4 & 396 & 761 \\
\hline & A5 & 124 & 245 \\
\hline & A6 & 76 & 136 \\
\hline \multirow[t]{6}{*}{ RsS3 / RsS32 } & $\mathrm{H} 1$ & 280 & 705 \\
\hline & $\mathrm{H} 2$ & 36 & 67 \\
\hline & $\mathrm{H} 3$ & 24 & 19 \\
\hline & $\mathrm{H} 4$ & 192 & 610 \\
\hline & H5 & 59 & 161 \\
\hline & $\mathrm{H} 6$ & 48 & 122 \\
\hline \multirow[t]{6}{*}{ RsS32 / RsS32 } & B1 & 9 & 10 \\
\hline & B2 & 12 & 7 \\
\hline & B3 & 16 & 13 \\
\hline & B4 & 0 & 0 \\
\hline & B5 & 0 & 0 \\
\hline & B6 & 8 & 7 \\
\hline
\end{tabular}

\section{Assembly of full-length genomic DNA sequences of S core genes in the $\mathrm{SC}$ radish}

To identify any defects in $S R K$ and $S C R / S P 11$ genes in the SC radish, full-length sequences of $S R K$ and $S C R / S P 11$ genes were analyzed. To identify the authentic SRK among duplicate SRK-like genes, full- 
length genomic DNA sequences of closely related SRK-like genes were obtained in this study. Using primers designed based on conserved regions among radish and three Brassica class II SRK genes (Supplementary Fig. 5), 5'S domain sequences of SRK were isolated. After obtaining sequences of the last exon7 using genome walking, a full-length SRK sequence was assembled by connecting 5' $S$ and 3' kinase domains through long PCR amplifications (Fig. 2). In addition, approximately 2-kb 5' sequences containing a putative promoter was obtained by genome walking, resulting in a 18,133-bp full-length sequence. Meanwhile, a 6,200-bp full-length sequence of another putative SRK gene was obtained using genome walking PCRs with primers designed based on dissimilar sequences between two putative SRK genes (Fig. 2).

Since partial $S$ domain sequences of the larger putative $S R K$ gene containing a large-sized 11,865-bp intron 3 were identical to those of the SRK-26 deposited in the GenBank (LC341218), the Shaplotype of the $S C$ radish might be identical to the RsS-26 haplotype designated by Haseyama et al. (2018). Indeed, nucleotide sequences of SLG of the SC radish were identical to those of SLG-26 (LC341241) of the RsS-26 haplotype. In addition, partial sequences identical to those of RsSCR-26 (LC325812) were amplified in the SC radish. However, additional 552-bp full-length SCR/SP11 gene sharing $91.0 \%$ sequence identities with RSSCR-26 was obtained by genome walking in the SC radish, implying that both SRK and SCR/SP11 genes might be duplicated in the RsS-26 haplotype. The novel $S C R / S P 11$ gene was closely related to other known SCR/SP11 genes (Supplementary Fig. 6). This novel gene was designated as RsSCR-26-2. Its sequence was deposited into GenBank under the accession number of MZ383800. The full-length RsSCR26-2 contained intact exons. Following the unified nomenclature suggested by Haseyama et al. (2018), the RsS3 haplotype was renamed as RsS-26 to avoid confusion.

Coding sequences of two putative SRK genes were also intact without any premature stop codons. They shared $91.7 \%$ nucleotide sequence identities with each other. Since $S$ core gene sequences were identical to those of the RsS-26 haplotype, the large and small putative SRK genes were designated as RsSRK-26-1 and RsSRK-26-2, respectively. Full-length sequences of both SRKs were deposited into GenBank under accession numbers of MZ383801 and MT241389, respectively. The phylogenetic tree of radish SRK genes showed that RsSRK-26-1 and RsSRK-26-2 were closely related to each other (Fig. 3). Similar to other class II SRK genes, the RSSRK-26-1 gene contained a large-sized intron 3. However, a relatively small intron 3 was identified in the RsSRK-26-2 gene (Supplementary Fig. 7), suggesting that the RsSRK-26-1 gene might be the genuine $S R K$ in the RsS-26 haplotype.

\section{Identification of a critical mutation responsible for the radish SC phenotype}

Interestingly, a transposable element-like sequence was found in the large-sized intron 3 of RsSRK-26-1 (Fig. 2). A 4,146-bp intact open reading frame (ORF) was identified in the transposable element-like sequence. Five typical domains of long terminal repeat (LTR)-retrotransposon were identified in the polyprotein region (Fig. 2). Since the integrase (INT) domain was positioned upstream of the reverse transcriptase (RT) domain, this LTR-retrotransposon belonged to Copia superfamily (Wicker et al. 2007). 
Target site duplication (TSD) of 4-bp ('GGAC') was found at flanking regions of this element. LTR sequences of 284-bp in length positioned at both ends were perfectly identical to each other. This novel LTR-retrotransposon was designated as RsCopia1.

Since the ORF encoding a polyprotein was intact and both LTR sequences were identical to each other, RsCopia 1 was assumed to be recently transposed into the RsSRK-26-1 gene. To investigate whether all RsS-26 haplotypes contained RsCopia 1 in the SRK gene, three breeding lines (DBRL294-2, DBR2085 and DBR2086) found to contain RsS-26 haplotypes in the previous study (Kim and Kim 2019) were analyzed. Results of PCR amplification and sequencing showed no RsCopia 1 insertion in any of these three breeding lines (Fig. 4A). Except for the RsCopia 1 insertion, only two single nucleotide polymorphisms (SNPs) were identified in the intron 3 between normal and mutant RsSRK-26-1 alleles. Nucleotide sequence of the normal allele was deposited into GenBank with accession number of MT241388.

Unlike SC JNUR1537 containing the mutant SRK allele, three breeding lines harboring normal SRK alleles showed SI phenotypes (Fig. 4C). Significantly reduced numbers of pods and seeds were produced in these three SI breeding lines (Fig. 4C, Supplementary Fig. 8). Transcripts of the mutant RsSKR-26-1 were not detected in the SC radish compared with the normal RSSRK-26-1 in three SI breeding lines (Fig. 4B). There was only a single SNP in putative promoter regions between normal and mutant RsSRK-26-1 alleles. These results suggest that transposition of RsCopia 1 in the RsSRK-26-1 gene might be responsible for the inactivation of $S R K$ and the resulting SC phenotype.

\section{Discussion}

\section{Identification of duplicate SRK-like genes in the radish RsS- 26 haplotype}

Full-length genomic DNA sequences of duplicated putative SRK and SCR/SP11 genes were obtained from the radish RsS-26 haplotype in this study (Fig. 2). Duplication of $S$ core genes including SCR/SP11 and SRK and its effects on SI responses have been reported in Brassica rapa (Takada et al. 2005, 2017) and Leavenworthia alabamica (Chantha et al. 2013), a member of Brassicaceae family. Takada et al. (2017) have demonstrated that duplicated SUI1 and PUI1 genes corresponding to SRK and SCR/SP11, respectively, control intraspecific unilateral incompatibility in $B$. rapa. In the case of Leavenworthia, a novel SI system might have evolved from paralogs ( $L a L a / 2$ and LaSCRL) of SRK and SCR/SP11 genes after loss of the original Slocus, which is common in Arabidopsis, Brassica, and Leavenworthia (Chantha et al. 2013). The phylogenetic tree indicated that duplication of SRK homologs in the radish RsS-26 haplotype occurred more recently than that in SUI1 and LaLal2 genes (Fig. 3).

However, it was unlikely that duplication was very recent since significant sequence and length polymorphisms existed between duplicated SRKs (Fig. 2). Since duplicate SCR/SP11 genes were also identified, the entire $S$ core region might be duplicated in the RsS-26 haplotype. Alternatively, two separate $S$ core regions might have been merged by homologous recombination-mediated translocation. Further 
studies are needed to elucidate the exact duplication event and effects of $S$ core region duplication. Isolation of full-length $S$ core regions of the RsS-26 haplotype might provide a clue to resolve these issues.

As shown in B. rapa (Takada et al. 2005, 2017) and Leavenworthia (Chantha et al. 2013), SI responses in the Brassicaceae family are complex processes. However, they are flexible enough to adopt duplicate paralogous $S R K$ and $S C R / S P 11$ pairs and restore SI systems after losing the original Slocus under sufficient selection pressure. Multigene family of SRK homologs in Brassicaceae (Cock et al. 1995; Suzuki et al. 1997; Pastuglia et al. 1997; Kai et al. 2001) represents a potential source of such plastic evolution of SI systems. In radish, a total of 61 SRK homologs have been identified from two draft genome sequences (Kim and Kim 2018). Further studies such as functional characterization of duplicate SRK and $S C R / S P 11$ genes and analysis of their effect on the strength of SI responses are needed to determine implications of $S$ core region duplication in the evolution of SI systems in Brassicaceae family. The radish $R s S-26$ haplotype harboring duplicate $S$ core genes represent a valuable material for such studies in the future.

\section{Identification of a radish breeding line showing a SC phenotype and its application in radish breeding}

A radish showing a SC phenotype was identified in this study. To the best of our knowledge, this is the first study to report an SC radish. Among Brassica species, several SC mutants have been previously reported. In Brassica rapa, SC mutants have been identified from two cultivars, Yellow Sarson (Fujimoto et al. 2006a) and Dahuangyoucai (Zhang et al. 2013), containing similar mutant Shaplotypes. Another SC phenotype induced by gene conversion from $S L G$ to $S R K$ has been reported (Fujimoto et al. 2006b). In addition, an SC B. rapa has been artificially developed by silencing of SCR/SP11 using RNAi (Jung et al. 2012). In the case of $B$. oleracea, deletion of exon 1 and 2 of SRK is responsible for an SC phenotype (Nasrallah et al. 1994). Recently, eight quantitative trait loci (QTLs) controlling a SC phenotype have been identified in an inbred line of B. oleracea (Xiao et al. 2019).

Duplicate $S R K$-like genes were identified from the SC radish in this study. Some evidences indicated that the large-sized RSSRK-26-1 might be a genuine SRK. First of all, transcription of RsSRK-26-1 was inactivated in the SC radish in contrast to three other SI breeding lines containing normal RSSRK-26-1. These results showed a direct relationship between SI phenotypes and RsSRK-26-1. In addition, the RSSRK-26-1 gene contained a large-sized intron 3 as shown in other Brassica class II SRK genes (Supplementary Fig. 7). Further functional studies are needed to clarify exact roles of both duplicate SRK genes. Since the RsSRK-26-2 gene contained intact exons and its transcripts were more abundant than those of RsSRK-26-1 (Fig. 4B), further functional characterization of RSSRK-26-2 might be an intriguing topic. 
An intact LTR-retrotransposon was identified in the large-sized intron 3 of $R S S R K-26-1$ of the SC radish in this study. Since this element was transposed into an intronic region, this insertion might not have any effect on transcription of SRK. Transcripts of RSSRK-26-1 were not detected in the mutant allele. Because there was only one single SNP in approximately $2.0 \mathrm{~kb}$ putative promoter regions between mutant and normal RSSRK-26-1 alleles, insertion of RsCopia 1 might be responsible for blockage of transcription. DNA methylation of promoter regions of RsSRK-26-1 is assumed to be induced by transposition of RsCopia 1, although further functional analyses are required. DNA methylation is known to be involved in silencing of transposable elements in plants (Bartels et al. 2018). Similarly, transcripts of the SRK gene are not detected in SC Yellow Sarson probably due to insertion of an LTR-retrotransposon in the intron 1, although there is no critical mutation in their promoter regions (Fujimoto et al. 2006a). In another case, reduced expression of the FLC gene is caused by insertion of an LTR-retrotransposon in the first intron (Michaels et al. 2003).

The SC phenotype observed in this study was apparent compared with phenotypes of SI breeding lines and $F_{2}$ individuals. The $S C$ phenotype was detected in $F_{1}$ and heterozygous $F_{2}$ individuals, suggesting that SC was dominant over SI in this population. This result indicates that the class II RsS-26 haplotype is dominant over a novel class II RsS32 haplotype. Despite a conspicuous SC phenotype, there were significant variations of pod and seed numbers produced by SC plants in this study. In addition to effects of minor modifying genes, environmental effects might play a significant role in the expression of SC phenotypes. High temperature is known to cause breakdown of SI phenotypes in Brassicaceae (Yamamoto et al. 2019). However, variations observed in this study might be largely derived from growth conditions where single plants were covered with mesh cages in the greenhouse. Large variations might result from such inferior growth conditions. When SC plants were grown in open field conditions, seed settings were significantly improved (Supplementary Fig. 9).

The $\mathrm{SC}$ radish identified in this study would be a valuable material for radish $\mathrm{F}_{1}$ hybrid breeding. Due to unstable SI phenotypes of some inbred lines, inadvertent self-pollination of maternal lines of $F_{1}$ hybrids frequently can result in a low genetic purity of $\mathrm{F}_{1}$ cultivars. For this reason, male-sterility has replaced $\mathrm{SI}$ systems as a more stable genetic emasculation tool. Although male-sterility is used for $F_{1}$ hybrid breeding, propagation of inbred parental lines should be performed by self-pollination of parental lines using high concentrations of $\mathrm{CO}_{2}$. However, if the $\mathrm{SC}$ phenotype is introgressed to parental lines, such expensive treatment with $\mathrm{CO}_{2}$ might be unnecessary. Regarding fixed varieties, the SC phenotype might greatly improve seed yields. Taken together, the SC radish identified in this study will become an important material for radish breeding programs.

\section{Declarations}

\section{Acknowledgments}

The authors thank Ji-hwa Heo, Jeong-An Yoo, and Su-jeong Kim for their dedicated technical assistance. 


\section{Authors' contribution}

So-Hyeon Bong and Ganghee Cho performed experiments and drafted the manuscript. Dong-Seon Kim performed phenotypic analyses. Sunggil Kim organized and coordinated this research project and edited the final manuscript.

\section{Funding}

This research was supported by Korea Institute of Planning and Evaluation for Technology in Food, Agriculture and Forestry (IPET) through a Golden Seed Project (Center for Horticultural Seed Development, No 213007-05-5-SBB10) and a grant from the Next-Generation BioGreen 21 Program (Plant Molecular Breeding Center No. PJ011034).

\section{Ethics approval}

All experiments were performed in compliance with current laws of the Republic of Korea.

\section{Consent for publication}

Not applicable.

\section{Competing interests}

The authors declare no competing interest.

\section{References}

1. Bartels A, Han Q, Nair P, Stacey L, Gaynier H, Mosley M, Huang QQ, Pearson JK, Hsieh T, An YC, Xiao W (2018) Dynamic DNA methylation in plant growth and development. Int J Mol Sci 19:2144

2. Boyes DC, Chen C, Tantikanjana T, Esch JJ, Nasrallah JB (1991) Isolation of a second S-LocusRelated cDNA from Brassica oleracea: genetic relationships between the Slocus and two related loci. Genetics 127:221-228

3. Brace J, Ockendon DJ, King GJ (1993) Development of a method for the identification of $S$ alleles in Brassica oleracea based on digestion of PCR-amplified DNA with restriction endonucleases. Sex Plant Reprod 6:133-138

4. Brace J, Ryder CD, Ockendon DJ (1994) Identification of S-alleles in Brassica oleracea. Euphytica 80:229-234

5. Castresana J (2000) Selection of conserved blocks from multiple alignments for their use in phylogenetic analysis. Mol Biol Evol 17:540-552

6. Chantha S, Herman AC, Platts AE, Vekemans X, Schoen DJ (2013) Secondary evolution of a selfincompatibility locus in the Brassicaceae genus Leavenworthia. PLoS Biol 11:e1001560 
7. Claessen H, Keulemans W, Van de Poel B, De Storme N (2019) Finding a Compatible Partner: SelfIncompatibility in European Pear (Pyrus communis); Molecular Control, Genetic Determination, and Impact on Fertilization and Fruit Set. Front Plant Sci 10:407

8. Cock JM, Stanchev B, Delorme V, Croy RRD, Dumas C (1995) SLR3: a modified receptor kinase gene that has been adapted to encode a putative secreted glycoprotein similar to the $S$ locus glycoprotein. Mol Gen Genet 248:151-161

9. Curtis IS (2011) Genetic engineering of radish: current achievement and future goals. Plant Cell Rep 30:733-744

10. Doyle JJ, Doyle JL (1987) A rapid DNA isolation procedure for small quantities of fresh leaf tissue. Phytochem Bull 19:11-15

11. Franceschi P, Dondini L, Sanzol J (2012) Molecular bases and evolutionaly dynamics of selfincompatibility in the Pyrinae (Rosaceae). J Exp Bot 63:4015-4032

12. Fujimoto R, Sugimura T, Fukai E, Nishio T (2006a) Suppression of gene expression of a recessive SP11/SCR allele by an untranscribed SP11/SCR allele in Brassica self-incompatibility. Plant Mol Biol 61:577-587

13. Fujimoto R, Sugimura T, Nishio T (2006b) Gene conversion from SLG to SRK resulting in selfcompatibility in Brassica rapa. FEBS Lett 580:425-430

14. Hall TA (1999) BioEdit: a user-friendly biological sequence alignment editor and analysis program for Window 95/98/NT. Nucl Acids Symp Ser 41:95-98

15. Haseyama Y, Kitashiba H, Okamoto S, Tonouchi E, Sakamoto K, Nishio T (2018) Nucleotide sequence analysis of Slocus genes to unify Shaplotype nomenclature in radish. Mol Breed 38:116

16. Igic B, Lande R, Kohn JR (2008) Loss of self-incompatibility and its evolutionary consequences. Int J Plant Sci 169:93-104

17. Jeong Y, Kim N, Ahn BO, Oh M, Chung W, Chung H, Jeong S, Lim K, Hwang Y, Kim G, Baek S, Choi S, Hyung D, Lee S, Sohn S, Kwon S, Jin M, Seol Y, Chae WB, Choi KJ, Park B, Yu H, Mun J (2016) Elucidating the triplicated ancestral genome structure of radish based on chromosome-level comparison with the Brassica genomes. Theor Appl Genet 129:1357-1372

18. Jung H, Jung H, Ahmed NU, Park J, Kang K, Hur Y, Lim Y, Nou I (2012) Development of selfcompatible B. rapa by RNAi-mediated Slocus gene silencing. PloS One 7:e49497

19. Kai N, Suzuki G, Watanabe M, Isogai A, Hinata K (2001) Sequence comparisons among dispersed members of the Brassica $S$ multigene family in an $S^{9}$ genome. Mol Genet Genomics 265:526-534

20. Kim D, Jung J, Choi Y, Kim S (2016) Development of a system for Slocus haplotyping based on the polymorphic SLL2 gene tightly linked to the locus determining self-incompatibility in radish (Raphanus sativus L.). Euphytica 209:525-535

21. Kim D, Kim S (2018) Identification of the Slocus core sequences determining self-incompatibility and $S$ multigene family from draft genome sequences of radish (Raphanus sativus L.). Euphytica 214:16 
22. Kim D, Kim S (2019) Development of a new Slocus haplotyping system based on three tightly linked genes in the $S$ locus controlling self-incompatibility in radish (Raphanus sativus L.). Sci Hortic 243:70-77

23. Kumar S, Stecher G, Li M, Knyaz C, Tamura K (2018) MEGA X: Molecular evolutionary genetics analysis across computing platforms. Mol Biol Evol 35:1547-1549

24. Li J, Cocker JM, Wright J, Webster MA, McMullan M, Dyer S, Swarbreck D, Caccamo M, van Oosterhout C, Gilmartin PM (2016) Genetic architecture and evolution of the Slocus supergene in Primula vulgaris. Nat Plants 2:16188

25. Lim SH, Cho HJ, Lee SJ, Cho YH, Kim BD (2002) Identification and classification of S haplotypes in Raphanus sativus by PCR-RFLP of the S locus glycoprotein (SLG) gene and the S locus receptor kinase (SRK) gene. Theor Appl Genet 104:1253-1262

26. Michaels SD, He Y, Scortecci KC, Amasino RM (2003) Attenuation of FLOWERING LOCUS C activity as a mechanism for the evolution of summer-annual flowering behavior in Arabidopsis. Proc Natl Acad Sci USA 100:10102-10107

27. Muñoz-Sanz JV, Zuriaga E, Cruz-García F, McClure B, Romero C (2020) Self-(In)compatibility systems: target traits for crop-production, plant breeding, and biotechnology. Front Plant Sci 11:195

28. Nasrallah JB, Kao TH, Goldberg ML, Nasrallah ME (1985) A cDNA clone encoding an S-locus-specific glycoprotein from Brassica oleracea. Nature 318:263-267

29. Nasrallah JB, Nishio BT, Nasrallah ME (1991) The self-incompatibility genes of Brassica: expression and use in genetic ablation of floral tissues. Ann Rev Plant Physiol Plant Mol Biol 42:393-422

30. Nasrallah JB, Rundle SJ, Nasrallah ME (1994) Genetic evidence for the requirement of the Brassica $S$-locus receptor kinase gene in the self-incompatibility response. Plant J 5:373-384

31. Nishio T, Kusaba M, Sakamoto K, Ockendon DJ (1997) Polymorphism of the kinase domain of the Slocus receptor kinase gene (SRK) in Brassica oleracea L. Theor Appl Genet 95:335-342

32. Nishio T, Kusaba M, Watanabe M, Hinata K (1996) Registration of Salleles in Brassica campestris L by the restriction fragment sizes of SLGs. Theor Appl Genet 92:388-394

33. Nishio T, Sakamoto K, Yamaguchi J (1994) PCR-RFLP of Slocus for identification of breeding lines in cruciferous vegetables. Plant Cell Rep 13:546-550

34. Nou IS, Watanabe M, Isogai A, Hinata K (1993) Comparison of Salleles and S-glycoproteins between two wild populations of Brassica campestris in Turkey and Japan. Sex Plant Reprod 6:79-86

35. Ockendon DJ (2000) The Sallele collection of Brassica oleracea. Acta Hort 539:79-86

36. Okamoto S, Sato Y, Sakamoto K, Nishio T (2004) Distribution of similar self-incompatibility (S) haplotypes in different genera, Raphanus and Brassica. Sex Plant Reprod 17:33-39

37. Pastuglia M, Roby D, Dumas C, Cock JM (1997) Rapid induction by wounding and bacterial infection of an S gene family receptor-like kinase gene in Brassica oleracea. Plant Cell 9:49-60

38. Sakamoto K, Kusaba M, Nishio T (2000) Single-seed PCR-RFLP analysis for the identification of S haplotypes in commercial $F_{1}$ hybrid cultivars of broccoli and cabbage. Plant Cell Rep 19:400-406 
39. Sato K, Nishio T, Kimura R, Kusaba M, Suzuki T, Hatakeyama K, Ockendon DJ, Satta Y (2002) Coevolution of the Slocus genes SRK, SLG and SCR/SP11 in Brassica oleracea and B. Rapa. Genetics 162:931-940

40. Schopfer CR, Nasrallah ME, Nasrallah JB (1999) The male determinant of self-incompatibility in Brassica. Science 286:1697-1700

41. Seo I, Kim J, Moon J, Kim S (2020) Construction of a linkage map flanking the / locus controlling dominant white bulb color and analysis of differentially expressed genes between dominant white and red bulbs in onion (Allium cepa L.). Euphytica 216:97

42. Silva NF, Goring DR (2001) Mechanisms of self-incompatibility in flowering plants. Mol Life Sci 58:1988-2007

43. Singh PK, Tripathi SK, Somani KV (2001) Hybrid seed production of radish (Raphanus sativus L.). J New Seeds 3:51-58

44. Sobotka R, Sáková L, Curn V (2000) Molecular mechanisms of self-incompatibility in Brassica. Curr Issues Mol Biol 2:103-112

45. Stone SL, Goring DR (2001) The molecular biology of self-incompatibility systems in flowering plants. Plant Cell Tiss Org 67:93-114

46. Suzuki G, Watanabe M, Kai N, Matsuda N, Toriyama K, Takayama S, Isogai A, Hinata K (1997) Three members of the Smultigene family are linked to the Slocus of Brassica. Mol Gen Genet 256:257-264

47. Takada Y, Murase K, Shimosato-Asano H, Sato T, Nakanishi H, Suwabe K, Shimizu KK, Lim YP, Takayama S, Suzuki G, Watanabe M (2017) Duplicated pollen-pistil recognition loci control intraspecific unilateral incompatibility in Brassica rapa. Nat Plants 3:17096

48. Takada Y, Nakanowatari T, Sato J, Hatakeyama K, Kakizaki T, Ito A, Suzuki G, Shiba H, Takayama S, Isogai A, Watanabe M (2005) Genetic analysis of novel intra-species unilateral incompatibility in Brassica rapa (syn campestris) L. Sex Plant Reprod 17:211-217

49. Takasaki T, Hatakeyama K, Suzuki G, Watanabe M, Isogai A, Hinata K (2000) The Sreceptor kinase determines self-incompatibility in Brassica stigma. Nature 403:913-916

50. Takayama S, Isogai A (2003) Molecular mechanism of self-recognition in Brassica selfincompatibility. J Exp Bot 54:149-156

51. Takayama S, Shiba H, Iwano M, Shimosato H, Che F, Kai N, Watanabe M, Suzuki G, Hinata K, Isogai A (2000) The pollen determinant of self-incompatibility in Brassica campestris. Proc Natl Acad Sci USA 97:1920-1925

52. Tantikanjana T, Nasrallah ME, Nasrallah JB (2010) Complex networks of self-incompatibility signaling in the Brassicaceae. Curr Opin Plant Biol 13:520-526

53. Tarutani Y, Shiba H, Iwano M, Kakizaki T, Suzuki G, Watanabe M, Isogai A, Takayama S (2010) Transacting small RNA determines dominance relationships in Brassica self-incompatibility. Nature 466:983-986 
54. Watanabe M, Suwabe K, Suzuki G (2012) Molecular genetics, physiology and biology of selfincompatibility in Brassicaceae. Proc Jpn Acad Ser B 88:519-535

55. Wheeler MJ, de Graaf BHJ, Hadjiosif N, Perry RM, Poulter NS, Osman K, Vatovec S, Harper A, Franklin FCH, Franklin-Tong VE (2009) Identification of the pollen self-incompatibility determinant in Papaver rhoeas. Nature 459:992-995

56. Wicker T, Sabot F, Hua-Van A, Bennetzen JL, Capy P, Chalhoub B, Flavell A, Leroy P, Morgante M, Panaud O, Paux E, SanMiguel P, Schulman AH (2007) A unified classification system for eukaryotic transposable elements. Nat Rev Genet 8:973-982

57. Xiao Z, Han F, Hu Y, Xue Y, Fang Z, Yang L, Zhang Y, Liu Y, Li Z, Wang Y, Zhuang M, Lv H (2019) Overcoming cabbage crossing incompatibility by the development and application of selfcompatibility-QTL- specific markers and genome-wide background analysis. Front Plant Sci 1:189

58. Yamamoto M, Nishimura K, Kitashiba H, Sakamoto W, Nishio T (2019) High temperature causes breakdown of $S$ haplotype-dependent stigmatic self-incompatibility in self-incompatible Arabidopsis thaliana. J Exp Bot 70:5745-5751

59. Yasuda S, Wada Y, Kakizaki T, Tarutani Y, Miura-Uno E, Murase K, Fujii S, Hioki T, Shimoda T, Takada Y, Shiba H, Takasaki-Yasuda T, Suzuki G, Watanabe M, Takayama S (2016) A complex dominance hierarchy is controlled by polymorphism of small RNAs and their targets. Nat Plants 3:16206

60. Yasui Y, Mori M, Aii J, Abe T, Matsumoto D, Sato S, Hayashi Y, Ohnishi O, Ota T (2012) S-LOCUS EARLY FLOWERING 3 is exclusively present in the genomes of short-styled buckwheat plants that exhibit heteromorphic self-incompatibility. PLoS One 7:e31264

61. Zhang X, Ma C, Yin D, Zhu W, Gao C, Zhang J, Fu T (2013) Characterization of Shaplotype in a new self-compatible Brassica rapa cultivar Dahuangyoucai. Czech J Genet Plant Breed 49:57-163

\section{Figures}
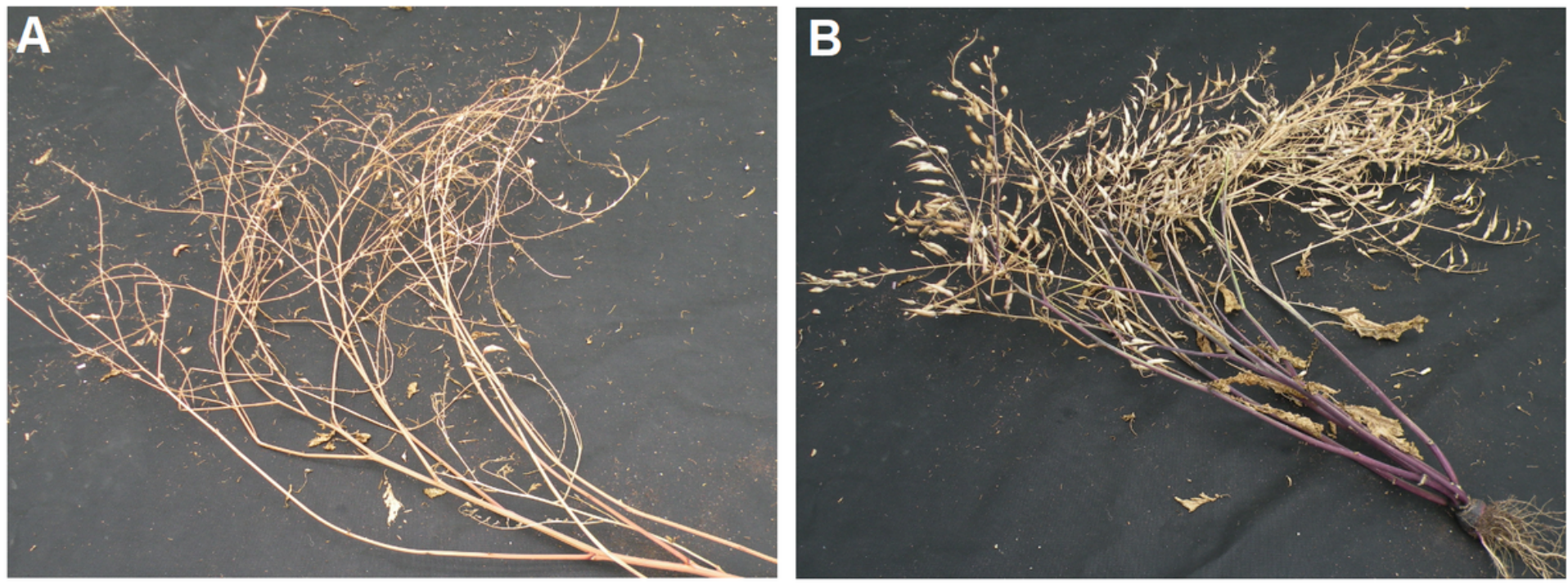

Figure 1 
Phenotypes of self-incompatible and self-compatible radish breeding lines at the stage of seed harvest. A. A self-incompatible breeding line, JNUR1123, B. A self-compatible breeding line, JNUR1537.

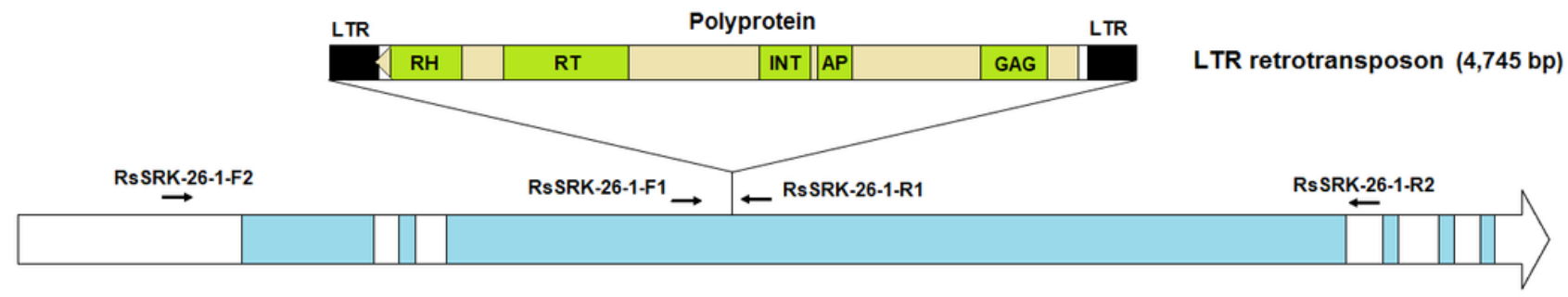

RSSRK-26-1

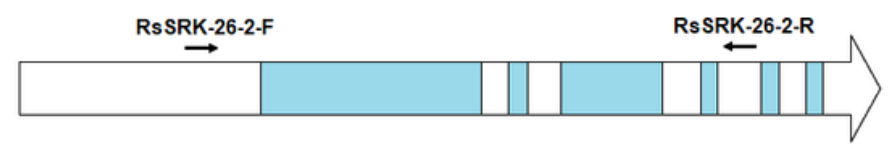

RsSRK-26-2

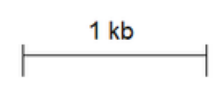

\section{Figure 2}

Genomic DNA structures of duplicated putative SRK genes in the radish RsS3 (RsS-26) haplotype. Empty and blue-colored boxes indicate exons and introns, respectively. Arrow-shaped boxes indicate 5'-to-3' direction. A rectangular box above the RsSRK-26-1 indicates a transposed LTR-retrotransposon. Arrowshaped box in the rectangular box indicates an ORF encoding a polyprotein and the 5'-to-3' direction. LTR: long terminal repeat, RH: RNase H, RT: Reverse transcriptase, INT: Integrase, AP: Aspartic proteinase, GAG: Capsid protein. Horizontal arrows indicate positions of primer binding sites. 


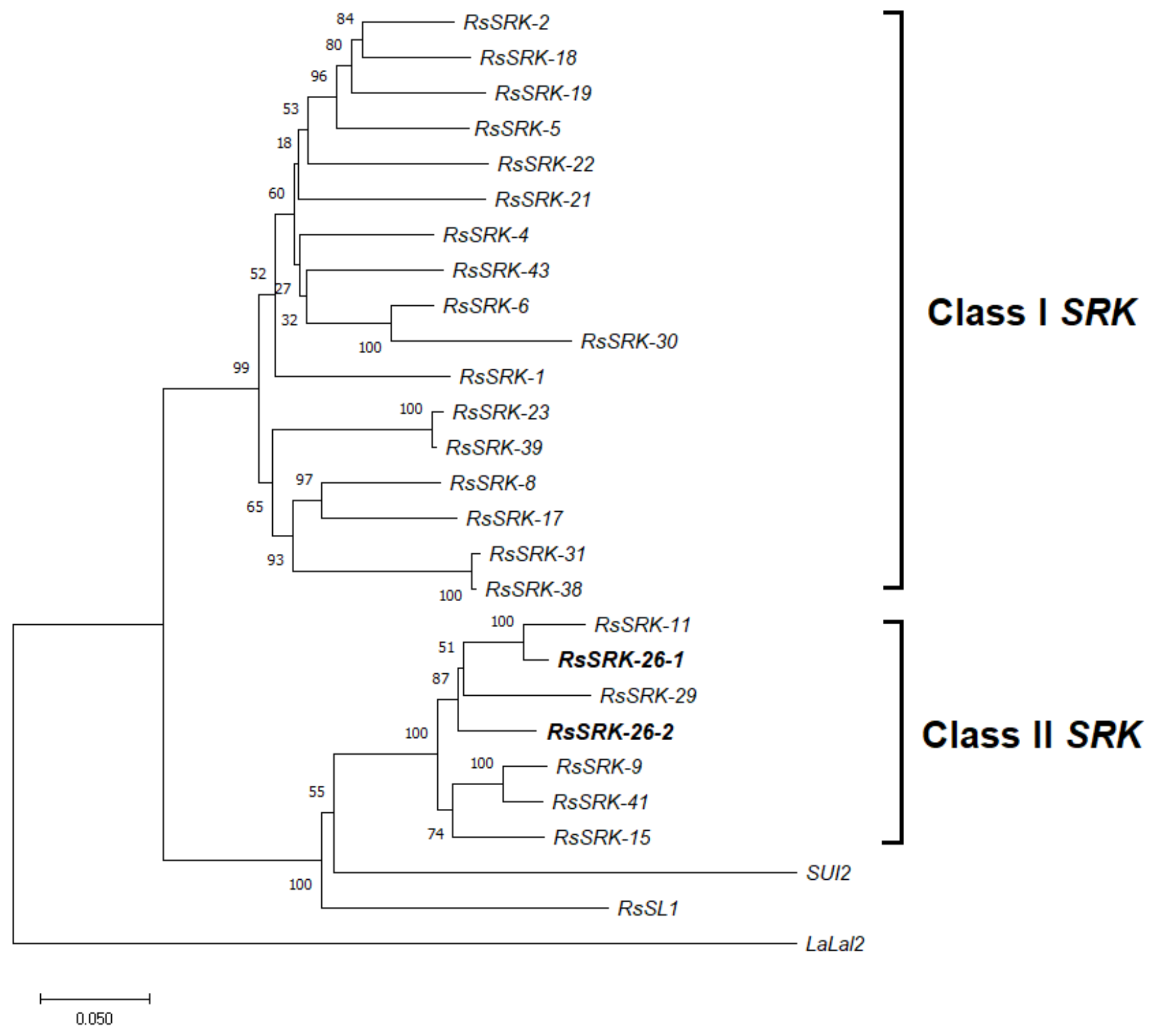

Figure 3

Phylogenetic relationship of S domain sequences of radish SRK genes. The tree was constructed with nucleotide sequences. Duplicate putative SRK genes positioned in the RsS3 (RsS-26) haplotype are shown in boldface. The RsSL1 is a radish SRK-homologous gene, which is most closely related to SRK genes (Kim and Kim 2018). SUI1 (GenBank accession: LC088714) and LaLal2 (GenBank accession: KC981242) are duplicated SRK genes identified from Brassica rapa (Takada et al. 2005, 2017) and Leavenworthia alabamica (Chantha et al. 2013), respectively. 
A
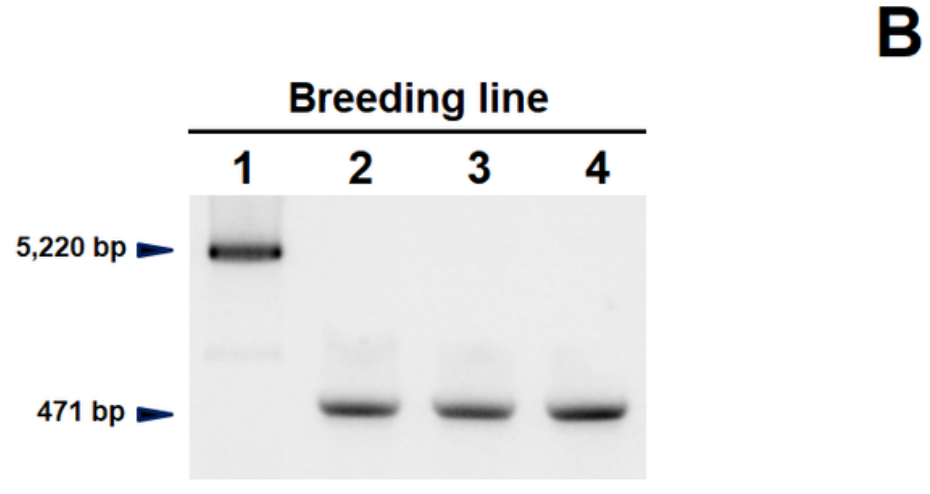

\section{\begin{tabular}{cccc}
\multicolumn{4}{c}{ Breeding line } \\
\hline 1 & 2 & 3 & 4
\end{tabular}}

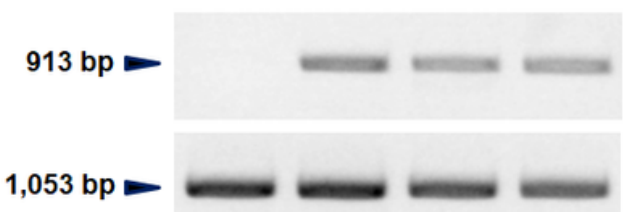

RsSRK-26-1

RsSRK-26-2

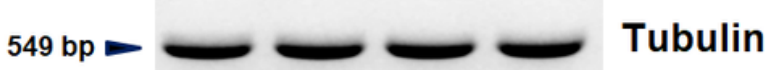

C
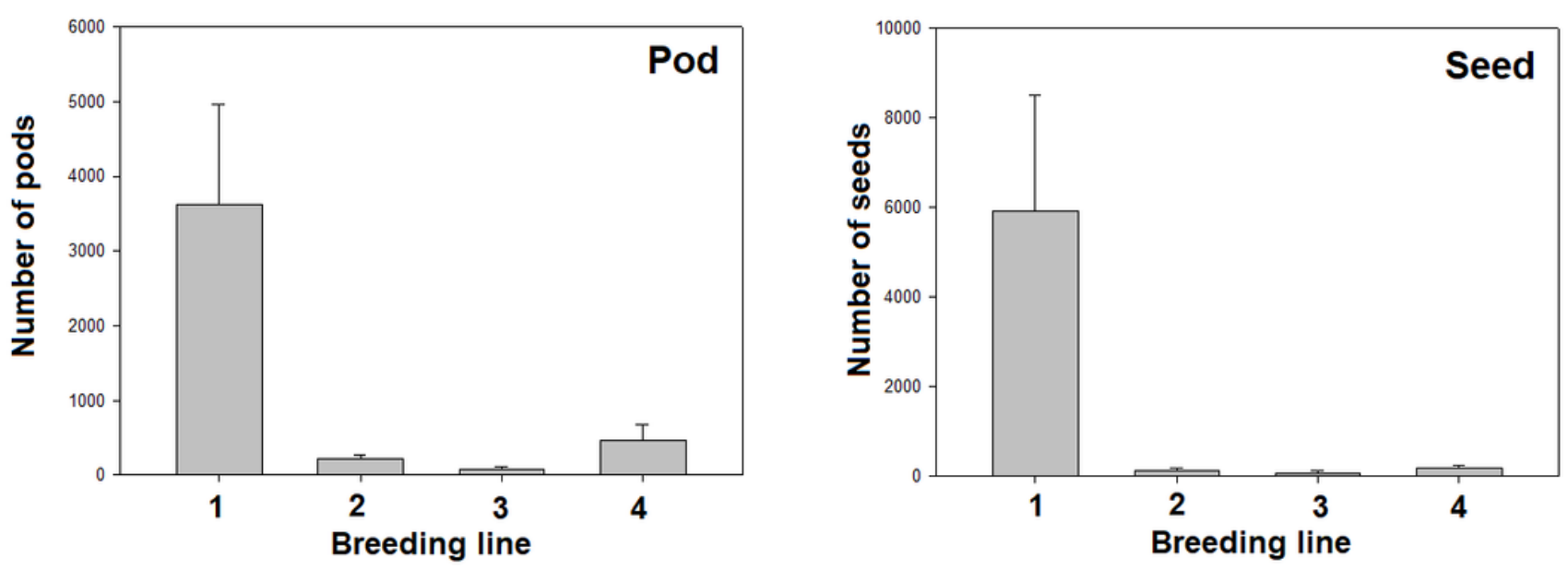

Figure 4

Comparison of breeding lines containing mutant and normal RsSRK-26-1 alleles. A. PCR products of RsSRK-26-1 amplified using a primer pair flanking the LTR-retrotransposon. B. RT-PCR products of RsSRK26-1 and RsSRK26-2 amplified from cDNAs of floral tissues. C. Comparison of pod and seed numbers produced from four breeding lines. Averages of four plants are shown in graphs. 1: JNUR1537, 2: DBRL294-2, 3: DBR2085, 4: DBR2086.

\section{Supplementary Files}

This is a list of supplementary files associated with this preprint. Click to download.

- SupplementaryFig.1.SLL2tree.tif

- SupplementaryFig.2.SP6tree.tif

- SupplementaryFig.3.SRKtree.tif

- SupplementaryFig.4.HRMmarkers.tif 
- SupplementaryFig.5.SRKalignment.tif

- SupplementaryFig.6.SCRtree.tif

- SupplementaryFig.7.FulllengthSRKs.tif

- SupplementaryFig.8.SlandSCphenotypes.tif

- SupplementaryFig.9.SCradishinthefield.tif

- SupplementaryTable1.Primersequences.xIsx 